\title{
Analysis of frozen orbits for solar sails
}

\author{
J. P. S. Carvalho, R. Vilhena de Moraes, \\ Instituto de Ciência e Tecnologia, UNIFESP, \\ São José dos Campos - SP, Brazil \\ E-mail: jeanfeg@gmail.com, rodolpho.vilhena@gmail.com, \\ A. Elipe, E. Tresaco, \\ Centro Universitario de la Defensa de Zaragoza, and \\ Grupo de Mecánica Espacial-IUMA, Universidad de Zaragoza, Spain. \\ E-mail: elipe@unizar.es, \\ A. F. B. A. Prado \\ INPE-Divisão de mecânica espacial e controle \\ São José dos Campos - SP, Brasil \\ E-mail:prado@dem.inpe.br
}

\begin{abstract}
The use of propellantless propulsion systems like solar sails in the recent days has attracted the interest for scientific missions. The influence of the radiation pressure on the sail creates an additional force to the dynamics of the problem, and this force which must be taken into account since it may modify greatly the behavior of the orbits. In this communication, we will see how the radiation pressure affects frozen orbits around a planet when the spacecraft is a solar sail, thus on the spacecraft the acting forces are the gravitational field of the planet, the gravitational perturbation due to the Sun (third body effect) and the radiation pressure on the sail.
\end{abstract}

keywords: Celestial mechanics, solar sail, frozen orbit, third-body perturbation.

\section{Introduction}

The use of propellantless propulsion systems like solar sails in the recent days has attracted the interest for scientific missions. Besides their obvious advantages as a propulsion system, solar sails are also considered for having "artificial" equilibria [3] for some observation missions, or to have "artificial" synchronous orbits, etc [12]. In [14] the authors consider displaced periodic orbits at linear order in the circular restricted Earth-Moon system, where the third massless body is a solar sail. In [2] the existence of artificial equilibrium points for a generalized sail is investigated. [10] shows that the solar sail is used to reach a geocentric sun-synchronous frozen orbit. This type of orbit combines the characteristics of sun-synchronous orbits and frozen orbits.

\section{Equations of motion}

We will base our work on previous results we obtained. Thus, in $[6,7,8]$ we found frozen orbits around Europa when the third body attraction (Jupiter) and in the gravity field of Europa zonal and tesseral harmonics are considered. By means of a Lie-transform, we average twice the Hamiltonian in order to reduce the problem to only one degree of freedom system, and thus have a surface depending on three variables, namely semi-major axis, eccentricity and inclination, which points will correspond to a frozen orbit. 
In this work, we use the same model, just replacing Europa by an inner planet, the third body by the Sun, and adding an extra term to the Hamiltonian, the one corresponding to the radiation pressure

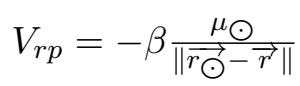

where the script $\odot$ stand for the Sun, $\mu_{\odot}$ is the Sun's gravitational parameter, $\vec{r}$ and $\vec{r} \odot$ are the position of spacecraft and Sun with respect to the planet and $\beta<1$ is a constant associated to the sail. With this, we obtain atlases of families of frozen orbits depending on the parameter $\beta$.

The $R_{2}$ term of the disturbing function due to the $P_{2}$ term (Legendre polynomial) can be written in the form [13]:

$$
R_{2}=\frac{\xi^{2} \mu_{\odot}}{a_{\odot}}\left(\frac{r}{a}\right)^{2}\left(\frac{a_{\odot}}{r_{\odot}}\right)^{3} P_{2}(\cos S)
$$

where $S$ is the angle between radius vectors. The artificial satellite is considered as a point mass particle in a three-dimensional orbit with osculating orbital elements: $a$ (semi-major axis), $e$ (eccentricity), $i$ (inclination), $g$ (argument of the periapsis), $h$ (longitude of the ascending node) and $n$ is the mean motion.

Here,

$$
\xi=\frac{a}{a_{\odot}}
$$

where $a_{\odot}$ is the semi-major axis of the Sun.

The disturbing body (Sun) is moving on a circular orbit. Using the relation between the angle $S$ and the true anomaly $f$ of the satellite, we get ([4])

$$
\cos (S)=\alpha \cos (f)+\beta \sin (f)
$$

where $f$ is the true anomaly of the artificial satellite. The coefficients $\alpha$ and $\beta$ are given for [4].

Using the known relationships from the celestial mechanics and Eqs. (2), (4), we average the equations according to the eccentric anomalies to eliminate short-period terms. Thus, we obtain the disturbing potential expanded up to the second order in a small parameter $(\xi)$.

The long-period disturbing potential using the double-averaged method can be written in the form:

$$
R_{2 S}=\frac{-1}{8 a_{\odot}^{3}} \mu_{\odot} a^{2}\left(15(\cos (g))^{2}(\cos (i))^{2} e^{2}-15(\cos (g))^{2} e^{2}-12(\cos (i))^{2} e^{2}-3(\cos (i))^{2}+9 e^{2}+1\right)
$$

Now using Eq. (1) and after some algebraic manipulations, we get

$$
R_{2 S \text { Sail }}=-\beta R_{2 S}
$$

where $\beta$ is the sail lightness number. The latter is a parameter that characterizes the performance of a solar sail. For a more refined mathematical model of the thrust provided by a non ideal solar sail the reader is referred to [12]. As the sail acceleration is small when compared to the gravitational acceleration of the Sun, in the following, is assumed to be less than one.

Combining the Eqs. (5) and (6), we obtain

$$
R_{2}=R_{2 S}+R_{2 S \text { Sail }}
$$

Thus, the potential is written in the form 


$$
\begin{aligned}
R_{2}= & \frac{-1}{8 a \odot^{3}}(1-\beta) \times \\
& \mu_{\odot} a^{2}\left(15(\cos (g))^{2}(\cos (i))^{2} e^{2}-15(\cos (g))^{2} e^{2}-12(\cos (i))^{2} e^{2}-3(\cos (i))^{2}+9 e^{2}+1\right)
\end{aligned}
$$

Since we assume that all perturbations are of the same order, and we only apply a first order Lie-transform, the obtained frozen orbits will be frozen within some limits, that is to say, the initial conditions thus obtained will correspond to some quasi-frozen orbits. By means of a numerical correction method $[11,9,1]$, we will refine these initial conditions to have frozen orbits for a wide time span.

For the perturbations due to nonuniform distribution of mass of the planet is taken into account the simplified model presented by [8]. Thus, considering the equatorial plane of the Earth as the reference plane, we obtain the disturbing potential given by [8]

$$
R_{J 2}=-\frac{1}{4} \frac{\epsilon n^{2}\left(-2+3 \sin ^{2}(i)\right)}{\left(1-e^{2}\right)^{3 / 2}}
$$

where $\epsilon=J_{2} R_{E}^{2}$ and $\mu$ is the gravitational constant of the Earth and $R_{E}$ is the equatorial radius of the Earth.

$$
R_{J 3}=-\frac{3}{8} \frac{e \epsilon_{1} n^{2} \sin (i)\left(-4+5 \sin ^{2}(i)\right) \sin (g)}{\left(1-e^{2}\right)^{5 / 2} a}
$$

where $\epsilon_{1}=J_{3} R_{E}^{3}$.

\section{Results}

This work is in process, therefore a preliminary result will just be presented here. We show a diagram of the eccentricity versus inclination to the value of the parameter $\beta$ equal to 0.02 and argument of periapsis equal to 90 degrees as shown in Fig. 1. Note that the orbit with lowest altitude $(a=45000 \mathrm{~km})$ presents greater effect on the variation of the eccentricity of that the orbit with higher altitude $(a=65000 \mathrm{~km})$. This is because the $J_{2}$ and $J_{3}$ terms which represent the non-spherical shape of the central body and produce greater effect for low-altitude orbits. The value of $g=90$ degrees is to freeze the orbit (see [5]). Frozen orbits are orbits that keep the periapsis and the eccentricity constant. Fig. 2 shows two curves for different values of the argument periapsis. When $g=45$ degrees some values of the inclination have a discontinuity. For $g=30$ degrees the eccentricity showed less variation than for $g=45$ degrees.

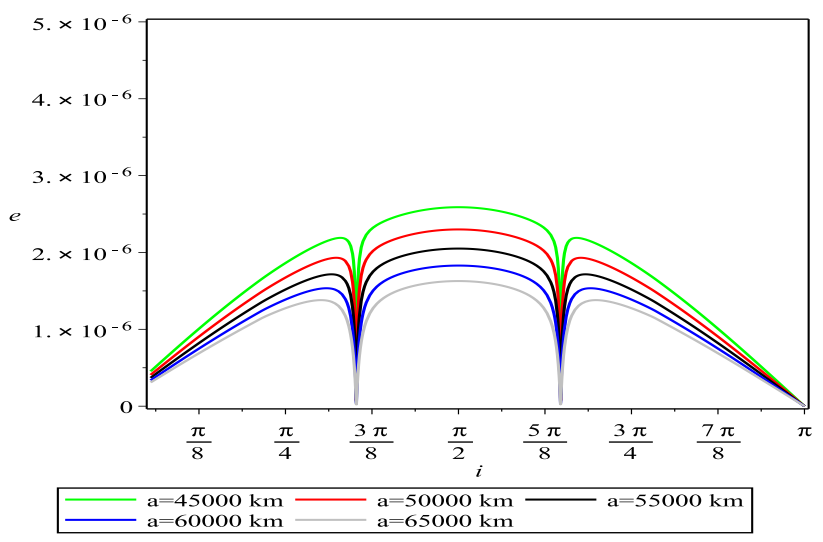

Figura 1: Diagram of the eccentricity versus inclination. $\beta=0.02, g=90^{\circ}$. 


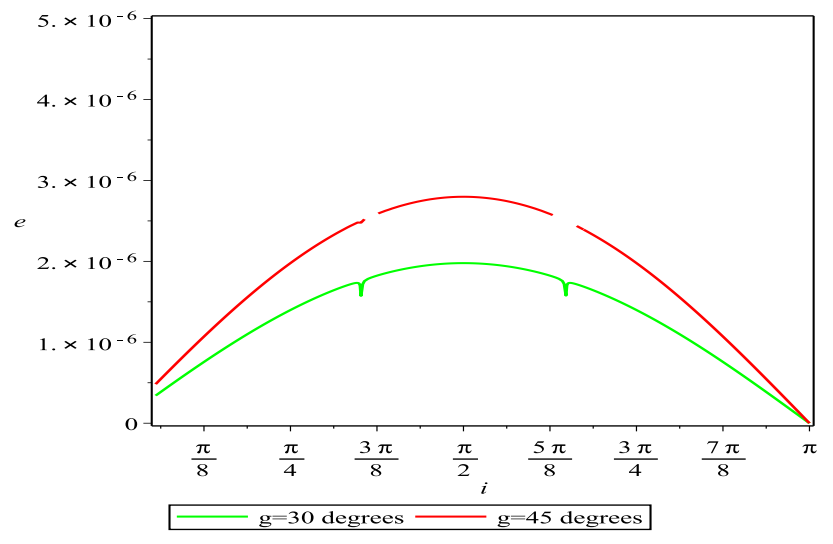

Figura 2: Diagram of the eccentricity versus inclination. $\beta=0.02, a=30000 \mathrm{~km}$.

\section{Conclusions}

To analyze the behavior of a satellite equipped with a solar sail into orbit around a planet (Earth) in this work, preliminary, is taken into account the perturbation of the third body (the Sun), the non-spherical shape of the planet and by the thrust provided by a solar sail. We show a diagram of the eccentricity versus inclination for different values of semi-major axis and argument of pericentre for the $\beta$ value equal to 0.02 . From these figures we can obtain the values of the eccentricity and inclination where frozen orbits can be found.

In continuation of this work we present numerical simulations for various trajectories taking into account the initial conditions of the eccentricity and inclination obtained in the diagram $e \times i$ in search of frozen orbits. A more detailed analysis of the effect of solar sail will be performed to improve the accuracy of the developed equations. We also intend to take into account the perturbation due to atmospheric drag.

\section{Acknowledgements}

The Authors are grateful to FAPESP (Foundation to Support Research in São Paulo State) under the contracts $N^{\circ}$ 2011/05671-5 and 2012/21023-6, SP-Brazil, CNPq (National Council for Scientific and Technological Development) - Brazil for contracts 304700/2009-6, 303070/2011-0 and CAPES.

\section{Referências}

[1] Abad, A., Elipe, A., Tresaco E. Analytical Model to Find Frozen Orbits for a Lunar Orbiter, Journal of Guidance, Control, and Dynamics. 32 (3), 888-898, 2009.

[2] Aliasi, G., Mengali, G., Quarta, A. A., Artificial equilibrium points for a generalized sail in the circular restricted three-body problem Celest Mech Dyn Astr 110, 343-368, 2011.

[3] Bombardelli, C., and Pelaez, J., On the stability of artificial equilibrium points in the circular restricted three-body problem, Celestial Mechanics and Dynamical Astronomy 109, 13-26, 2011.

[4] Broucke, R. A. Long-Term Third-Body Effects via Double Averaging, Journal of Guidance, Control, and Dynamics, 26 (1), 272003.

[5] Carvalho, J. P. dos S., Vilhena de Moraes, R., Prado, A. F. B. A. Some Orbital Characteristics of Lunar Artificial Satellites, Celest. Mech. Dyn. Astron. 108 (4), 371-388, 2010 . 
[6] Carvalho, J. P. S., Elipe, A., Vilhena de Moraes, R., Prado, A. F. B. A., Low-altitude, near-polar and near-circular orbits around Europa, Advances in Space Research 49 (5), 994-1006, 2012.

[7] Carvalho, J. P. S., Mourão, D. C., Elipe, A., Vilhena de Moraes, R., Prado, A. F. B. A., Frozen orbits around the Europa satellite, Iternational Journal of Bifurcation and Chaos 22 (10), 1250240, 2012.

[8] Carvalho, J. P. S., Vilhena de Moraes, R., Prado, A. F. B. A., Dynamics of Articial Satellites around Europa. Mathematical Problems in Engineering, v.2013, Article ID 182079, 2013.

[9] Deprit, A., Canonical transformations depending on a small parameter, Celestial Mechanics 1, 12-30, 1969.

[10] Gong S. P., Li J. F., Baoyin H. X., Simo, J., A new solar sail orbit. Sci China Tech Sci, $55(3), 848-855,2012$.

[11] Hori, G., The Motion of a Hyperbolic Artificial Satellite around the Oblate Earth, The Astronomical Journal, Vol. 66, No. 6, pp. 258-263, 1961.

[12] McInnes, C. R., "Solar Sailing: Technology, Dynamics and Mission Applications", Springer-Praxis Series in Space Science and Technology, Springer-Verlag, 1999.

[13] Murray, C. D., Dermott S. F. Solar System Dynamics. Cambridge: Cambridge University Press, 592p, 1999.

[14] Simo, J. and McInnes, C.R. Displaced solar sail orbits: dynamics and applications. In: 20th AAS/AIAA Space Flight Mechanics Meeting, 14-17 Feb 2010, San Diego, California, 2010. 